\title{
Adaptive Control of Uncertain Hamiltonian Multi-Input Multi-Output Systems : With Application to Spacecraft Control
}

\author{
Hyungjoo Yoon and Brij N. Agrawal
}

\begin{abstract}
A novel adaptive control law for nonlinear Hamiltonian Multi-Input Multi-Output (MIMO) systems with uncertain parameters in the actuator modeling as well as the inertia and/or the Coriolis and centrifugal terms is developed. The physical properties of the Hamiltonian systems are effectively used in the control design and the stability analysis. The number of the parameter estimates is significantly lowered as compared to the conventional adaptive control methods. A smooth projection algorithm is applied to keep the parameter estimates inside a singularity-free region. The developed control scheme is applied for attitude control of a spacecraft with both the inertia and the actuator uncertainties.
\end{abstract}

\section{INTRODUCTION}

We consider a multi-input multi-output (MIMO) nonlinear Hamiltonian system represented by the second-order differential equation

$$
\begin{gathered}
H(\mathbf{q}) \ddot{\mathbf{q}}+C(\mathbf{q}, \dot{\mathbf{q}}) \dot{\mathbf{q}}+\mathbf{g}(\mathbf{q})=F \\
F=D(\mathbf{q}) \mathbf{u}
\end{gathered}
$$

where $\mathbf{q} \in \mathbb{R}^{n}$ is the generalized coordinates vector, $H \in \mathbb{R}^{n \times n}$ is the (symmetric positive definite) inertia matrix, $C \dot{\mathbf{q}}$ is a nonlinear vector of Coriolis and centripetal forces, and $\mathbf{g} \in \mathbb{R}^{n}$ is the gravity vector. $F \in \mathbb{R}^{n}$ is the generalized force and is generated by a control input vector $\mathbf{u} \in \mathbb{R}^{m}$ and the actuator matrix $D \in \mathbb{R}^{n \times m}$. For full tracking control, it is generally required that $n \leq m$ and $D$ has full row rank. Expressing dynamics of systems in the form of Eq. (1), rather than the state-space form, has several advantages. Equation (1) can be easily derived by applying Lagrange's equation, and its form is so general that it can represent various kinds of dynamic systems, such as a multilink robot manipulator [1], [2] and a spacecraft [3]-[5], etc. In addition, there is a physical property that the matrix $\dot{H}-2 C$ is skewsymmetric. This property is extremely useful in designing advanced control schemes.

Suppose now that the system matrices have uncertainties in their parameters and can be expressed as

$$
\begin{gathered}
H\left(\mathbf{q}, \Theta_{s}\right)=H^{n}(\mathbf{q})+H^{\Delta}\left(\mathbf{q}, \Theta_{s}\right) \\
C\left(\mathbf{q}, \dot{\mathbf{q}}, \Theta_{s}\right)=C^{n}(\mathbf{q}, \dot{\mathbf{q}})+C^{\Delta}\left(\mathbf{q}, \dot{\mathbf{q}}, \Theta_{s}\right) \\
\mathbf{g}\left(\mathbf{q}, \Theta_{s}\right)=\mathbf{g}^{n}(\mathbf{q})+\mathbf{g}^{\Delta}\left(\mathbf{q}, \Theta_{s}\right) \\
D\left(\mathbf{q}, \Theta_{a}\right)=D^{n}(\mathbf{q})+D^{\Delta}\left(\mathbf{q}, \Theta_{a}\right)
\end{gathered}
$$

where the matrices with a superscript of ' $n$ ' are with their known nominal values, $\Theta_{a} \in \mathbb{R}^{p}$ is a vector of unknown

H. Yoon and B.N. Agrawal are with the Dept. of Mechanical and Astronautical Engineering, Naval Postgraduate School, Monterey, CA 93943, USA. Email : (See http://www.drake.googlepages.com), agrawalenps.edu bounded constant uncertainties in the actuator matrix $D$, and $\Theta_{s} \in \mathbb{R}^{q}$ is in the other system matrices/vector, $H, C$, and $\mathbf{g}$. We also assume that, by proper definition of the unknown parameters $\Theta_{s}$ and $\Theta_{a}$, the uncertain matrices $H^{\Delta}\left(\mathbf{q}, \Theta_{s}\right), C^{\Delta}\left(\mathbf{q}, \dot{\mathbf{q}}, \Theta_{s}\right), \mathbf{g}^{\Delta}\left(\mathbf{q}, \Theta_{s}\right)$ and $D^{\Delta}\left(\mathbf{q}, \Theta_{a}\right)$ are linearly dependent on $\Theta_{s}$, and $\Theta_{a}$, respectively.

Adaptive control for special cases where the actuator modeling does not have uncertainties, that is $D^{\Delta}=0$, has been intensively studied in the literature (see for instance Ref. [1]). However, adaptive control for more general cases with $D^{\Delta} \neq 0$ does not seem to have received much attention in the literature, even though this uncertainty may result in significant degeneration of controller performance. Ge [6] has derived an adaptive control law for multilink manipulator systems with uncertainties in the control input term, but the uncertainty must be in the input scalings, and thus the uncertainty matrix must be diagonal when represented in multiplicative form. (Or it can be said that $D^{\Delta}=\Delta D^{n}$ where $\Delta$ is a diagonal matrix.) Chang [7] has provided an adaptive, robust tracking control algorithm for nonlinear MIMO systems which is based on the "smooth projection algorithm," which has also been used in [8] and [9] for adaptive control of SISO systems. More recently, one of the authors [10], [11] has also provided an adaptive control scheme based on the smooth projection algorithm which is applied to spacecraft attitude tracking with uncertain misalignments/inertia of the actuator flywheels. However, these previous results [7], [10], [11] are based on purely mathematical approaches and do not exploit the useful physical properties of the Hamiltonian systems. More significantly, they considered MIMO systems represented by the differential equations without any terms in front of the highest derivative of the state variable vector, like the state-space form. Therefore, in their methods, Eq. (1) would need to be converted as

$$
\ddot{\mathbf{q}}=-H^{-1} C \dot{\mathbf{q}}-H^{-1} \mathbf{g}+H^{-1} D \mathbf{u},
$$

in which the uncertain matrices are multiplied to each other. Therefore, in order to design adaptive laws based on the linear dependency of uncertainties, their methods need a "over-parameterization", which means they need to estimate the combinations of the elements in $\Theta_{a}$ and $\Theta_{s}$, and thus the number of parameter estimates would significantly increase.

In the present paper, an adaptive control algorithm for the general case where the uncertain matrices in Eq. (2) are all nonzero is developed using the smooth projection algorithm, which keeps the parameter estimates inside a properly defined singular-free convex set. The proposed adaptive law exploits the physical properties of the Hamiltonion system 
and has a more compact form with a smaller number of parameter estimates. The proposed method is then applied for a spacecraft attitude control problem with inertia/actuator uncertainties. Finally, numerical examples with the spacecraft are provided to validate the proposed law.

\section{Adaptive Control LaW}

The first part of the derivation of the adaptive control law follows the standard design procedure for Hamiltonian systems in [1], [4]. Let us assume the desired trajectory $\mathbf{q}_{d}(t), \dot{\mathbf{q}}_{d}(t)$ and $\ddot{\mathbf{q}}_{d}(t)$ to be bounded. The tracking error vector is defined as $\tilde{\mathbf{q}} \triangleq \mathbf{q}-\mathbf{q}_{d}$ and a measure of tracking $\mathbf{s}$ and the reference velocity $\dot{\mathbf{q}}_{r}$ are defined as

$$
\mathbf{s} \triangleq \dot{\tilde{\mathbf{q}}}+\Lambda \tilde{\mathbf{q}}=\dot{\mathbf{q}}-\dot{\mathbf{q}}_{r}
$$

and

$$
\dot{\mathbf{q}}_{r} \triangleq \dot{\mathbf{q}}_{d}-\Lambda \tilde{\mathbf{q}}
$$

where the matrix $-\Lambda$ is assumed to be Hurwitz. Let $\hat{\Theta}_{*}$ be the parameter estimate vector and let $\tilde{\Theta}_{*} \triangleq \hat{\Theta}_{*}-\Theta_{*}$ be a parameter estimate error vector, when $*$ is $a$ or $s$.

As suggested by Slotine et. al. [1] from a physical insight that $\dot{\mathbf{q}}^{T} H \dot{\mathbf{q}}$ is the system's kinetic energy, the following Lyapunov function candidate is defined as

$$
V(t)=\frac{1}{2}\left[\mathbf{s}^{T} H \mathbf{s}+\tilde{\Theta}_{a}^{T} \Gamma_{a}^{-1} \tilde{\Theta}_{a}+\tilde{\Theta}_{s}^{T} \Gamma_{s}^{-1} \tilde{\Theta}_{s}\right]
$$

where $\Gamma_{a}, \Gamma_{s}$ are positive definite weighting matrices.

Differentiating $V(t)$ with respect to time and using the skew-symmetry property of the matrix $\dot{H}-2 C$ to replace the term $\frac{1}{2} \mathbf{s}^{T} \dot{H} \mathbf{s}$ with $\mathbf{s}^{T} C \mathbf{s}$, one can have the following expression:

$$
\dot{V}=\mathbf{s}^{T}\left(D \mathbf{u}-H \ddot{\mathbf{q}}_{r}-C \dot{\mathbf{q}}_{r}-\mathbf{g}\right)+\tilde{\Theta}_{a}^{T} \Gamma_{a}^{-1} \dot{\tilde{\Theta}}_{a}+\tilde{\Theta}_{s}^{T} \Gamma_{s}^{-1} \dot{\tilde{\Theta}}_{s} .
$$

When the actuator matrix $D$ is exactly known (i.e., $D^{\Delta}=0$ ) and has full row rank (i.e., $\operatorname{rank}(D)=\mathrm{n}$ ), one can easily design an adaptive control law using the methods proposed in the previous works [1].

However, since $D$ is assumed to contain unknown parameters as well as $H, C$ and $\mathbf{g}$, a novel control law is proposed as follows:

$$
\begin{gathered}
\left(D^{n}+\hat{D}^{\Delta}\right) \mathbf{u}=\left(\left(H^{n}+\hat{H}^{\Delta}\right) \ddot{\mathbf{q}}_{r}+\left(C^{n}+\hat{C}^{\Delta}\right) \dot{\mathbf{q}}_{r}\right. \\
\left.+\left(\mathbf{g}^{n}+\hat{\mathbf{g}}^{\Delta}\right)-K_{d} \mathbf{s}\right)
\end{gathered}
$$

where the matrices/vector with a 'hat' symbol are constructed using the parameter estimates $\hat{\Theta}_{a}$ and $\hat{\Theta}_{s}$ instead of the (unknown) actual parameters. $K_{d}$ is a gain matrix which is a positive definite. When the matrix $D^{n}+\hat{D}^{\Delta}$ is assumed to have full row rank, the (weighted) minimum norm solution is given by

$$
\begin{array}{r}
\mathbf{u}=\left(D^{n}+\hat{D}^{\Delta}\right)^{\dagger}\left(\left(H^{n}+\hat{H}^{\Delta}\right) \ddot{\mathbf{q}}_{r}+\left(C^{n}+\hat{C}^{\Delta}\right) \dot{\mathbf{q}}_{r}\right. \\
\left.+\left(\mathbf{g}^{n}+\hat{\mathbf{g}}^{\Delta}\right)-K_{d} \mathbf{S}\right)
\end{array}
$$

where $(\cdot)^{\dagger}$ denotes the (weighted) pseudo-inverse of a matrix [5], [11].
The control law (9) leads to

$$
\begin{aligned}
\dot{V}=\mathbf{s}^{T} & {\left[\left\{\left(D^{n}+\hat{D}^{\Delta}\right)-\tilde{D}^{\Delta}\right)\right\} \mathbf{u}-\left(H^{n}+H^{\Delta}\right) \ddot{\mathbf{q}}_{r}-\left(C^{n}+C^{\Delta}\right) \dot{\mathbf{q}}_{r} } \\
& \left.\quad-\left(\mathbf{g}^{n}+\mathbf{g}^{\Delta}\right)\right]+\tilde{\Theta}_{a}^{T} \Gamma_{a}^{-1} \dot{\tilde{\Theta}}_{a}+\tilde{\Theta}_{s}^{T} \Gamma_{s}^{-1} \dot{\tilde{\Theta}}_{s} \\
=\mathbf{s}^{T} & {\left[\left(H^{n}+\hat{H}^{\Delta}\right) \ddot{\mathbf{q}}_{r}+\left(C^{n}+\hat{C}^{\Delta}\right) \dot{\mathbf{q}}_{r}+\left(\mathbf{g}^{n}+\hat{\mathbf{g}}^{\Delta}\right)-K_{d} \mathbf{s}\right.} \\
& \left.\quad-\tilde{D}^{\Delta} \mathbf{u}-\left(H^{n}+H^{\Delta}\right) \ddot{\mathbf{q}}_{r}-\left(C^{n}+C^{\Delta}\right) \dot{\mathbf{q}}_{r}-\left(\mathbf{g}^{n}+\mathbf{g}^{\Delta}\right)\right] \\
& +\tilde{\Theta}_{a}^{T} \Gamma_{a}^{-1} \dot{\tilde{\Theta}}_{a}+\tilde{\Theta}_{s}^{T} \Gamma_{s}^{-1} \dot{\tilde{\Theta}}_{s} \\
=\mathbf{s}^{T} & {\left[\tilde{H}^{\Delta} \ddot{\mathbf{q}}_{r}+\tilde{C}^{\Delta} \dot{\mathbf{q}}_{r}+\tilde{\mathbf{g}}^{\Delta}-K_{d} \mathbf{s}-\tilde{D}^{\Delta} \mathbf{u}\right] } \\
& +\tilde{\Theta}_{a}^{T} \Gamma_{a}^{-1} \dot{\tilde{\Theta}}_{a}+\tilde{\Theta}_{s}^{T} \Gamma_{s}^{-1} \dot{\tilde{\Theta}}_{s} \\
=- & \mathbf{s}^{T} K_{d} \mathbf{s}+\mathbf{s}^{T}\left(\tilde{H}^{\Delta} \ddot{\mathbf{q}}_{r}+\tilde{C}^{\Delta} \dot{\mathbf{q}}_{r}+\tilde{\mathbf{g}}^{\Delta}\right)-\mathbf{s}^{T} \tilde{D}^{\Delta} \mathbf{u} \\
& +\tilde{\Theta}_{a}^{T} \Gamma_{a}^{-1} \tilde{\tilde{\Theta}}_{a}+\tilde{\Theta}_{s}^{T} \Gamma_{s}^{-1} \tilde{\tilde{\Theta}}_{s}
\end{aligned}
$$

Since the uncertainty matrices/vector are assumed to depend linearly on $\Theta$ 's, one can define known regressor matrices (in fact, row vectors) $Y_{s}=Y_{s}\left(\mathbf{q}, \dot{\mathbf{q}}, \dot{\mathbf{q}}_{r}, \ddot{\mathbf{q}}_{r}, \mathbf{s}\right) \in \mathbb{R}^{1 \times q}$ and $Y_{a}=Y_{a}\left(\mathbf{q}, \dot{\mathbf{q}}, \dot{\mathbf{q}}_{r}, \ddot{\mathbf{q}}_{r}, \mathbf{s}, \mathbf{u}\right) \in \mathbb{R}^{1 \times p}$ such that

$$
\mathbf{s}^{T}\left(H^{\Delta} \ddot{\mathbf{q}}_{r}+C^{\Delta} \dot{\mathbf{q}}_{r}+\mathbf{g}^{\Delta}\right)=Y_{s} \Theta_{s}
$$

and

$$
-\mathbf{s}^{T} D^{\Delta} \mathbf{u}=Y_{a} \Theta_{a}
$$

Notice that the definition of the regressor matrix $Y_{S}$ in Eq. (11) is slightly different from that in the previous works by Slotine [1], [3]. By including $\mathbf{s}$ in the regressor's definition, the size of the regressor $Y_{s}$ becomes smaller than that of Slotine's works where the regressor has a size of $n \times q$.

The time derivative of $V$ then becomes

$$
\dot{V}=-\mathbf{s}^{T} K_{d} \mathbf{s}+Y_{s} \tilde{\Theta}_{s}+Y_{a} \tilde{\Theta}_{a}+\tilde{\Theta}_{s}^{T} \Gamma_{s}^{-1} \dot{\hat{\Theta}}_{s}+\tilde{\Theta}_{a}^{T} \Gamma_{a}^{-1} \dot{\hat{\Theta}}_{a}
$$

and taking the adaptation laws of the parameter estimates to be

$$
\dot{\hat{\Theta}}_{s}=-\Gamma_{s} Y_{s}^{T}
$$

and

$$
\dot{\hat{\Theta}}_{a}=-\Gamma_{a} Y_{a}^{T}
$$

then yields

$$
\dot{V}(t)=-\mathbf{s}^{T} K_{d} \mathbf{s} \leq 0 .
$$

Using standard arguments in [1], [11] which use Barbalat's lemma, one can easily show that $\dot{V} \rightarrow 0$ and thus $\mathbf{s} \rightarrow 0$ as $t \rightarrow \infty$. This also implies the tracking error $\tilde{\mathbf{q}} \rightarrow 0$ as well.

\section{A. Smooth Projection Algorithm}

We previously used an assumption that the matrix $D^{n}+\hat{D}^{\Delta}$ has full row rank in deriving the adaptive control laws, Eqs.(9) ,(14) and (15). In general, for the full tracking control, the nominal matrix $D^{n}$ in general has full row rank. However, a drift of the parameter estimates $\hat{\Theta}_{a}$, governed by an update law (15), can result in $D^{n}+\hat{D}^{\Delta}$ losing rank. We will refer to this situation as a "singularity" of the steering law due to the adaptation. This singularity hinders the use of the derived control laws, so they need to be modified. 
If the nominal matrix $D^{n}$ has full row rank, and the true value of the parameter uncertainty $\Theta_{a}$ is bounded by a sufficiently small number, and the parameter estimate $\hat{\Theta}_{a}$ is also kept small, then the matrix $D^{n}+\hat{D}^{\Delta}$ will also have full row rank. To this end, we define the following two convex sets,

$$
\begin{gathered}
\Omega_{\Theta_{a}} \triangleq\left\{\Theta_{a} \in \mathbb{R}^{p} \mid\left\|\Theta_{a}\right\|^{2}<\beta\right\} \\
\hat{\Omega}_{\Theta_{a}} \triangleq\left\{\hat{\Theta}_{a} \in \mathbb{R}^{p} \mid\left\|\hat{\Theta}_{a}\right\|^{2}<\beta+\delta\right\}
\end{gathered}
$$

where $\beta>0$ and $\delta>0$ are known constants. Notice that $\Omega_{\Theta_{a}} \subset \hat{\Omega}_{\Theta_{a}}$. We make the following three assumptions.

- Assumption 1. The nominal value $D^{n}$ has full row rank of $n$.

- Assumption 2. The actual value $\Theta_{a}$ belongs to the set $\Omega_{\Theta_{a}}$.

- Assumption 3. If $\hat{\Theta}_{a} \in \hat{\Omega}_{\Theta_{a}}$, then $D^{n}+\hat{D}^{\Delta}$ is nonsingular.

These assumptions allow us to modify the adaptation law (15) by using the "smooth projection algorithm" as follows 1 :

$$
\dot{\hat{\Theta}}_{a}=\operatorname{Proj}\left(\hat{\Theta}_{a}, \Phi_{a}\right)
$$

where

$$
\Phi_{a} \triangleq-\Gamma_{a} Y_{a}^{T}
$$

and

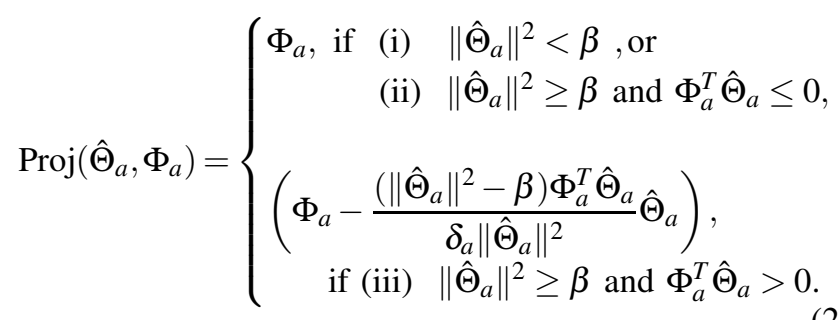

This adaptation law is identical to (15) in cases (i) and (ii), and switches smoothly to a new expression in case (iii). The projection operator $\operatorname{Proj}\left(\hat{\Theta}_{a}, \Phi_{a}\right)$ is locally Lipschitz in $\left(\hat{\Theta}_{a}, \Phi_{a}\right)$, thus the system has a unique solution defined for some time interval $[0, T), T>0$.

Proposition 1: Under Assumptions 1,2, and 3, the control law Eq. (9) along with the adaptation laws Eqs. (14) and (19) yields

$$
\dot{V} \leq-\mathbf{s}^{T} K_{d} \mathbf{s} \leq 0
$$

and

$$
\hat{\Theta}_{a}(t=0) \in \Omega_{\Theta_{a}} \Rightarrow \hat{\Theta}_{a}(t) \in \hat{\Omega}_{\Theta_{a}}, \quad \forall t \geq 0 .
$$

Proof: The proof is straightforward and therefore omitted here. It is similar with the proof in the author's previous works [11], [13].

From Proposition 1, one can conclude that, using the feedback control law (9) and the adaptation laws (14) and (19), $\tilde{\mathbf{q}} \rightarrow 0$ as $t \rightarrow \infty$ and $\left(D^{n}+\hat{D}^{\Delta}\right)$ will not lose rank, if

\footnotetext{
${ }^{1}$ The adaptation law is, in fact, only Lipschitz continuous, not continuously differentiable. The use of the term "smooth" is a slight misnomer in this context, but we use it here in accordance to prior usage in the literature. It should be noted that a new parameter projection operator which is $\mathscr{C}^{n}$ has been recently introduced in Ref. [12].
}

we choose the initial parameter guess $\hat{\Theta}_{a}(0)$ inside the set $\Omega_{\Theta_{a}}$. For instance, we may take $\hat{\Theta}_{a}(0)=0$.

It is also worth mentioning that the proposed adaptation law (19) has the additional benefit of keeping the parameter estimates from "bursting", which may happen when the persistency of excitation condition does not hold [14].

\section{Application to Spacecraft Attitude Control}

\section{A. Equations of Motion}

In this section, applying the proposed adaptive control scheme, we design an adaptive attitude tracking control law for a spacecraft. A cluster of Variable-Speed Control Moment Gyros (VSCMGs) with $N$ flywheels is used for the torque actuator. While a conventional Control Moment Gyro (CMG) keeps its flywheel spinning at a constant rate, a VSCMG - as its name implies - is essentially a single-gimbal CMG with the flywheel allowed to have variable speed. (See Refs. [10], [15] for more details and applications of VSCMGs.)

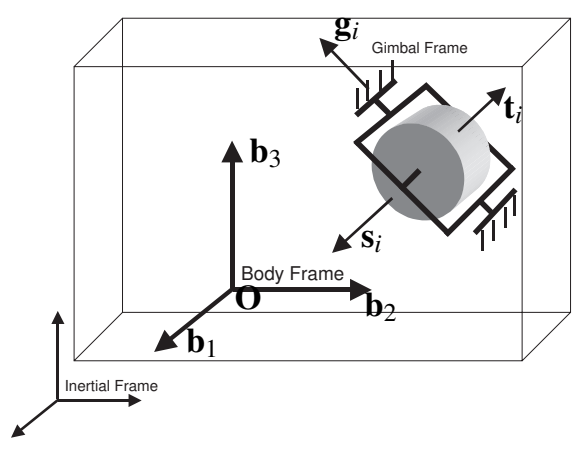

Fig. 1. Spacecraft Body with the $i$-th VSCMG.

Figure 1 shows a spacecraft with the $i$-th VSCMG, where $\mathbf{g}_{i}$ is (body-fixed) gimbal axis, $\mathbf{s}_{i}$ is spin axis, and $\mathbf{t}_{i} \triangleq \mathbf{g}_{i} \times$ $\mathbf{s}_{i}$ is transverse torque axis. The equations of motion of a spacecraft with VSCMGs are complicated as shown in the aforementioned references, but under assumptions which are standard in the literature [11], [13], they can be simplified as follows:

$$
J \dot{\omega}-\left[\mathbf{h}^{\times}\right] \omega+Q \mathbf{u}=0,
$$

and

$$
\mathbf{h}=J \omega+A_{s} I_{w} \Omega,
$$

where

$$
Q=\left[Q_{c m g}, Q_{r w}\right] \in \mathbb{R}^{3 \times 2 N},
$$

and where $Q_{c m g}(\gamma, \Omega)=A_{t} I_{w} \Omega^{d}, Q_{r w}(\gamma)=A_{s} I_{w}$, and the control input of this system is

$$
\mathbf{u}=\left[u_{1}, \cdots, u_{2 N}\right]^{T}=\left[\dot{\gamma}^{T}, \dot{\Omega}^{T}\right]^{T} \in \mathbb{R}^{2 N} .
$$

$J$ is the total moment of inertia of the spacecraft which is assumed to be constant ${ }^{2}, \omega$ is the body rate vector of the spacecraft, $\mathbf{h}$ is the total angular momentum of the spacecraft, $\gamma=\left[\gamma_{1}, \ldots, \gamma_{N}\right]^{T} \in \mathbb{R}^{N}$ and $\Omega=\left[\Omega_{1}, \ldots, \Omega_{N}\right]^{T} \in \mathbb{R}^{N}$ are vectors of gimbal angles and flywheel spinning speeds,

\footnotetext{
${ }^{2}$ In fact, $J$ is a function of $\gamma$ but the dependence is weak in general.
} 
respectively, and $I_{w}$ is a diagonal matrix with the inertias of VSCMGs flywheels. The skew-symmetric matrix $\left[\mathbf{v}^{\times}\right]$, for $\mathbf{v} \in \mathbb{R}^{3}$, represents the cross product operation. The matrices $A_{*} \in \mathbb{R}^{3 \times N}$ have as columns the gimbal $\left(\mathbf{g}_{i}\right)$, spin $\left(\mathbf{s}_{i}\right)$ and transverse $\left(\mathbf{t}_{i}\right)$ directional unit vectors expressed in the bodyframe, where $*$ is $\mathrm{g}, \mathrm{s}$ or $\mathrm{t}$. These matrices depend on the gimbal angles as follows

$$
\begin{aligned}
A_{g} & =A_{\mathrm{g} 0} \\
A_{s} & =A_{\mathrm{s} 0}[\cos \gamma]^{d}+A_{\mathrm{t} 0}[\sin \gamma]^{d} \\
A_{t} & =A_{\mathrm{t} 0}[\cos \gamma]^{d}-A_{\mathrm{s} 0}[\sin \gamma]^{d}
\end{aligned}
$$

where the $A_{* 0}$ 's denote the values of $A_{*}$ at $\gamma=0$. The symbol $\mathbf{x}^{d}$ denotes the diagonal matrix with elements the components of the vector $\mathbf{x}$, and $\cos \gamma \triangleq\left[\cos \gamma_{1}, \cdots, \cos \gamma_{N}\right]^{T}$ and $\sin \gamma \triangleq$ $\left[\sin \gamma_{1}, \cdots, \sin \gamma_{N}\right]^{T}$.

The modified Rodrigues parameters (MRPs) [16]-[18] are chosen to describe the attitude kinematics of the spacecraft. ${ }^{3}$ The kinematics in terms of the MRPs is given by

$$
\dot{\sigma}=G(\sigma) \omega
$$

where

$$
G(\sigma)=\frac{1}{2}\left(\mathbf{I}_{3}+\left[\sigma^{\times}\right]+\sigma \sigma^{T}-\left[\frac{1}{2}\left(1+\sigma^{T} \sigma\right)\right] \mathbf{I}_{3}\right)
$$

and $\mathbf{I}_{r}$ is the $r \times r$ identity matrix.

As suggested in Refs. [1] and [5], we combine the kinetic equation (24) and the kinematic equations (31) into one second-order system as follows:

$$
H(\sigma) \ddot{\sigma}+C(\sigma, \dot{\sigma}) \dot{\sigma}=D(\sigma) \mathbf{u}
$$

where

$$
\begin{aligned}
H(\sigma) & =G^{-T} J G^{-1}, \\
C(\sigma, \dot{\sigma}) & =-G^{-T} J G^{-1} \dot{G} G^{-1}-G^{-T}\left[\left(R(\sigma) \mathbf{h}^{I}\right)^{\times}\right] G^{-1}, \\
D(\sigma) & =-G^{-T} Q .
\end{aligned}
$$

$R(\sigma)$ is a rotational matrix from the inertial frame to the body frame, and $\mathbf{h}^{I}$ is the total angular momentum of a spacecraft expressed in the inertial frame which is conserved to be constant if there is no external torque applied to the spacecraft. Therefore, $\mathbf{h}=R(\sigma) \mathbf{h}^{I}$.

Notice that the equation of motion (33) has the form of (1) with the gravitational term $\mathbf{g}=0$. Moreover, it can be easily shown that the matrix $\dot{H}-2 C$ is skew-symmetric [1].

\section{B. Adaptive Attitude Tracking Control}

Suppose that there are uncertainties in $D$ as well as $J$. We assume that the exact values of the initial axis directions of VSCMGs actuator at $\gamma=0$ are unknown. This can happen when the VSCMGs are installed with small misalignments and/or the measure of gimbal angles has constant unknown bias. In addition, $\mathbf{h}^{I}$ is also unknown constant not only because of uncertain $J$ but also because of uncertain $A_{s}$.

\footnotetext{
${ }^{3}$ We hasten to point out that the use of the MRPs to describe the kinematics is done without loss of generality. Any other suitable kinematic description could have been used with the conclusions of the paper remaining essentially the same.
}

For most cases the effect of axes uncertainties on the overall system performance is not significant. However, for the case of flywheels used as "mechanical batteries" in an Integrated Power and Attitude Control System (IPACS) [5], [19], [20], even small misalignments of the flywheel axes can be detrimental. Flywheels for IPACS applications spin at high speeds and have large amounts of stored kinetic energy (and hence angular momentum). Precise attitude control requires proper momentum management, while minimizing spurious output torques. This can be achieved with the use (in the simplest scenario) of at least four flywheels, whose angular momenta have to be canceled or regulated with high precision. If the exact direction of the axes (hence the direction of the angular momenta) are not known with sufficient accuracy, large output torque errors will impact the attitude of the spacecraft.

The uncertain parameters in $H$ and $C$ can be defined as follows:

$\Theta_{s}=\left[\Delta j_{11}, \Delta j_{22}, \Delta j_{33}, \Delta j_{12}, \Delta j_{13}, \Delta j_{23}, \Delta h_{1}, \Delta h_{2}, \Delta h_{3}\right]^{T} \in \mathbb{R}^{9}$

where $\Delta j$ 's are the elements of $J^{\Delta} \triangleq J-J^{n}$ and $J^{n}$ is the nominal value of the actual inertia matrix $J$. Similarly, $\Delta h$ 's are elements of $\mathbf{h}^{I \Delta} \triangleq \mathbf{h}^{I}-\mathbf{h}^{I n}$. The uncertain parameters in $D$ are defined as

$$
\Theta_{a}=\left[\Theta_{t, 1}^{T}, \cdots, \Theta_{t, N}^{T}, \Theta_{s, 1}^{T}, \cdots, \Theta_{s, N}^{T}\right]^{T} \in \mathbb{R}^{6 N}
$$

where

$$
\Theta_{t, i} \triangleq \mathbf{t}_{i, 0}-\mathbf{t}_{i, 0}^{n}, \quad \Theta_{s, i} \triangleq \mathbf{s}_{i, 0}-\mathbf{s}_{i, 0}^{n}, \quad i=1, \cdots, N .
$$

and $\mathbf{t}_{i, 0}$ and $\mathbf{s}_{i, 0}$ are actual value of $\mathbf{t}_{i}$ and $\mathbf{s}_{i}$ at $\gamma_{i}=0$, respectively, and $\mathbf{t}_{i, 0}^{n}$ and $\mathbf{s}_{i, 0}^{n}$ are their nominal values. The total number of parameter estimates is then $6 N+9$. If one uses the methods in the previous works [7], [10], [11], [13], then the number of the estimates will be as much as $6 \times(6 N+3)$.

Exact mathematical expressions of the regressor vectors $Y_{s}$ defined in (11) can be easily obtained using symbolic math packages, and can be constructed from the measurements of $\sigma, \dot{\sigma}$, and the desired trajectories $\sigma_{d}, \dot{\sigma}_{d}, \ddot{\sigma}_{d}$. The regressor vector $Y_{a}$ defined in (12) can be obtained in the same way, but it is also possible to derive its mathematical expression by manipulating the matrices as follows:

$$
Y_{a}=\mathbf{s}^{T} G^{-T}\left[(M \mathbf{u})_{1} \mathbf{I}_{3}, \cdots,(M \mathbf{u})_{2 N} \mathbf{I}_{3}\right]
$$

where

$$
M \triangleq\left[\begin{array}{cc}
{[\cos \gamma]^{d}} & {[\sin \gamma]^{d}} \\
-[\sin \gamma]^{d} & {[\cos \gamma]^{d}}
\end{array}\right]\left[\begin{array}{cc}
I_{w} \Omega^{d} & 0 \\
0 & I_{w}
\end{array}\right]
$$

and $(\mathbf{x})_{i}$ is the $i$ th element of a vector $\mathbf{x}$. Then using the developed control scheme in Sec. II, one can design an adaptive attitude tracking control law for the spacecraft.

\section{NUMERICAL EXAMPLES}

Numerical examples for a satellite with a VSCMGs cluster are provided in this section to test the proposed adaptive control algorithm. A standard four-VSCMG pyramid configuration $(N=4)$ is utilized [20]. The parameters used for 
TABLE I

SiMULATION PARAMETERS AND GAINS

\begin{tabular}{ccc}
\hline Symbol & Value & Units \\
\hline \hline$\sigma(0)$ & {$[0,0,0]^{T}$} & - \\
$\omega(0)$ & {$[0,0,0]^{T}$} & $\mathrm{rad} / \mathrm{sec}^{T}$ \\
$\dot{\omega}(0)$ & {$[0,0,0]^{T}$} & $\mathrm{rad} / \mathrm{sec}^{2}$ \\
$\gamma(0)$ & {$[0,0,0,0]^{T}$} & $\mathrm{rad}$ \\
$\dot{\gamma}(0)$ & {$[0,0,0,0]^{T}$} & $\mathrm{rad} / \mathrm{sec}$ \\
$\Omega(0)$ & $10^{4} \times[2.5,3.5,3.5,3.0]^{T}$ & $\mathrm{rpm}$ \\
$I_{w}$ & $\operatorname{diag}\{2.0,2.0,2.0,2.0\}$ & $\mathrm{kg} \mathrm{m}^{2}$ \\
$K_{d}$ & $10^{3} \mathbf{I}_{3}$ & - \\
$\Lambda$ & $\mathbf{I}_{3}$ & - \\
$\beta$ & 0.01 & - \\
$\delta$ & 0.01 & - \\
\hline
\end{tabular}

the simulations are shown in Table I. Notice that the initial wheel speeds of the VSCMGs are set to 25,000 35,000 RPM, which are an order of magnitude larger than the speed of conventional CMGs, since the flywheels of VSCMGs used for IPACS in general need to spin very fast so that they are competitive against traditional chemical batteries. According to Ref. [21], even a higher speed than these vales is implementable, at least in a laboratory.

The nominal values of the axis directions at $\gamma=[0,0,0,0]^{T}$ are

$$
\begin{gathered}
A_{s 0}^{\mathrm{n}}=\left[\begin{array}{cccc}
0 & -1 & 0 & 1 \\
1 & 0 & -1 & 0 \\
0 & 0 & 0 & 0
\end{array}\right], \\
A_{t 0}^{\mathrm{n}}=\left[\begin{array}{cccc}
-0.5774 & 0 & 0.5774 & 0 \\
0 & -0.5774 & 0 & 0.5774 \\
0.8165 & 0.8165 & 0.8165 & 0.8165
\end{array}\right] .
\end{gathered}
$$

The (unknown) actual axis directions at $\gamma=0$ used in the present example are assumed as

$$
\begin{aligned}
& A_{s 0}=\left[\begin{array}{cccc}
-0.0072 & -0.9999 & -0.0072 & 0.9999 \\
0.9999 & 0.0143 & -0.9999 & 0.0057 \\
-0.0071 & 0.0071 & -0.0142 & -0.0117
\end{array}\right], \\
& A_{t 0}=\left[\begin{array}{cccc}
-0.5657 & -0.0024 & 0.5831 & 0.0061 \\
0.0018 & -0.5715 & -0.0157 & 0.5868 \\
0.8246 & 0.8206 & 0.8123 & 0.8097
\end{array}\right],
\end{aligned}
$$

which are obtained by rotating each nominal gimbal frame $\left(\mathbf{g}_{i}, \mathbf{s}_{i}, \mathbf{t}_{i}\right)$ with 1 degree about arbitrary direction. With these values, $\left\|\Theta_{a}\right\|^{2} \simeq 0.0015$. The nominal value of the spacecraft inertia matrix is

$$
J^{n}=\left[\begin{array}{ccc}
15000 & 3000 & -1000 \\
3000 & 6500 & 2000 \\
-1000 & 2000 & 12000
\end{array}\right] \quad \mathrm{kg} \mathrm{m}^{2}
$$

and the (unknown) actual inertia matrix is

$$
J=\left[\begin{array}{ccc}
18000 & 2400 & -1200 \\
2400 & 5200 & 1600 \\
-1200 & 1600 & 14400
\end{array}\right] \quad \mathrm{kg} \mathrm{m}^{2}
$$

which is obtained by adding/subtracting $20 \%$ of the nominal values. The reference trajectory is chosen so that the initial reference attitude is aligned with the body frame which is also aligned with the inertial frame, and the angular velocity of the reference attitude is chosen as

$$
\omega_{d}(t)=0.04\left(\sin \frac{2 \pi t}{400}, \sin \frac{2 \pi t}{300}, \sin \frac{2 \pi t}{200}\right)^{T} \mathrm{rad} / \mathrm{sec} .
$$

First, in order to show the effect of the misalignment of the axes of the VSCMG cluster and the uncertain inertia matrix, a simulation without adaptation was performed. Figure 2 shows the attitude tracking error (expressed with ' $3-2-1$ ' Euler angles) ${ }^{4}$ under control law with the adaptation gains $\Gamma_{a}$ and $\Gamma_{s}$ set to zero matrices. Since the flywheel speeds are very fast, there is large attitude tracking error without adaptation.

Next, another simulation was run with adaptation of the actuator uncertainty $\Theta_{a}$ only. The adaptation gains are set to $\Gamma_{a}=10 \mathbf{I}_{6 N}$ and $\Gamma_{s}=0$, and the resulting attitude error is shown in Fig. 3. There is significant performance improvement with the adaptation of $\Theta_{a}$ only, but tracking error with a magnitude of about 0.2 degree remains. On the other hand, Fig. 4 shows the attitude tracking error with adaptation of $\Theta_{s}$ only. The adaptation gains are $\Gamma_{a}=0$ and $\Gamma_{s}=\operatorname{diag}\left(10^{7} \mathbf{I}_{6}, 10^{5} \mathbf{I}_{3}\right)$. In fact, the control law in this scenario is almost identical with Slotine's method [1]. The attitude error is significantly attenuated using the adaptive controller, but there are again residual tracking errors with a magnitude of about 0.1 degree.

Finally, a simulation is performed with adaptation of both $\Gamma_{a}$ and $\Gamma_{s}$, that is $\Gamma_{a}=10 \mathbf{I}_{6 N}$ and $\Gamma_{s}=\operatorname{diag}\left(10^{7} \mathbf{I}_{6}, 10^{5} \mathbf{I}_{3}\right)$. Figure 5 shows the tracking performance is improved upon Slotine's control law. Figure 6 shows the time history of $\left\|\hat{\Theta}_{a}\right\|^{2}$. It is confirmed that $\left\|\hat{\Theta}_{a}\right\|^{2}$ does not drift more than $\beta+\delta=0.02$ owing to the smooth projection algorithm. As a result, the steering law (9) remains well-defined.

\section{CONCLUSIONS}

In this paper, we proposed an adaptive tracking control law for a nonlinear Hamiltonian MIMO dynamic system. The proposed control scheme has several significant improvements over the previous works in the literature. First, the proposed method can deal with uncertainties in the actuator terms which Slotine's method [1] does not deal with. The regressor matrix for adaptation of the uncertain inertia also has a smaller size than that of [1]. Second, the proposed method exploits the physical properties of the Hamiltonian systems and so the designed law is more compact than those in [7], [11], [13] which deal with actuator uncertainties but are derived based on purely mathematical approaches. Finally, the proposed method deals with the actuator uncertainties separately from the uncertain inertia/Coriolis/gravity terms. Therefore, it does not need over-parameterization to deal with both kinds of uncertainties at the same time, while [7], [11], [13] do.

The developed adaptive algorithm is shown to significantly improve the tracking performance in the application to the

\footnotetext{
${ }^{4}$ The use of Euler angles in the figures in done solely for the convenience of the reader who may not be familiar with the MRPs.
} 
spacecraft attitude control, but it still has room for improvement. For instance, while only 3 parameters are generally needed to express a misalignment of axis frame for one VSCMG, a total of 6 parameters are used in this method. Development of methods to reduce the number of estimated parameters would be extremely beneficial.

\section{REFERENCES}

[1] J. Slotine and W. Li, Applied Nonlinear Control. New Jersey: Prentice Hall, 1991.

[2] F. L. Lewis, C. T. Abdallah, and D. M. Dawson, Control of Robot Manipulators. New York, NY: Macmillan Publishing Company, 1993.

[3] J. J. E. Slotine and M. D. D. Benedetto, "Hamiltonian adaptive control of spacecraft," IEEE Transactions on Automatic Control, vol. 35, no. 7, pp. 848-852, July 1990.

[4] T. I. Fossen, "Comments on "hamiltonian adaptive control of spacecraft"," IEEE Transactions on Automatic Control, vol. 38, no. 4, pp. 671-672, April 1993.

[5] H. Yoon and P. Tsiotras, "Spacecraft adaptive attitude and power tracking with variable speed control moment gyroscopes," Journal of Guidance, Control, and Dynamics, vol. 25, no. 6, pp. 1081-1090, Nov.-Dec. 2002.

[6] S. S. Ge, "Adaptive control of robots having both dynamical parameter uncertainties and unknown input scalings," Mechatronics, vol. 6, no. 5, pp. 557-569, 1996.

[7] Y.-C. Chang, "An adaptive $\mathscr{H}_{\infty}$ tracking control for a class of nonlinear multiple-input-multiple-output (MIMO) systems," IEEE Transactions on Automatic Control, vol. 46, no. 9, pp. 1432-1437, Sep. 2001.

[8] J.-B. Pomet and L. Praly, "Adaptive nonlinear regulation : Estimation from the Lyapunov equation," IEEE Transactions on Automatic Control, vol. 37, no. 6, pp. 729-740, June 1992.

[9] H. K. Khalil, "Adaptive output feedback control of nonlinear systems represented by iuput-output models," IEEE Transactions on Automatic Control, vol. 41, no. 2, pp. 177-188, Feb. 1996.

[10] H. Yoon, "Spacecraft attitude and power control using variable speed control moment gyros," Ph.D. dissertation, Georgia Institute of Technology, Atlanta, GA., December 2004, (URL:http://hdl.handle.net/1853/4850).

[11] H. Yoon and P. Tsiotras", "Adaptive spacecraft attitude tracking control with actuator uncertainties," Journal of the Astronautical Sciences, submitted for publication.

[12] Z. Cai, M. de Queiroz, and D. Dawson, "A sufficiently smooth projection operator," IEEE Transactions on Automatic Control, vol. 51, no. 1, pp. 135-139, Jan. 2006.

[13] H. Yoon and P. Tsiotras, "Adaptive spacecraft attitude tracking control with actuator uncertainties," in AIAA Guidance, Navigation, and Control Conference, San Francisco, California, August 2005, aIAA2005-6392.

[14] B. D. O. Anderson, "Failures of adaptive control theory and their resolution," Communications in Information and Systems, vol. 5, no. 1, pp. 1-20, 2005.

[15] H. Schaub, S. R. Vadali, and J. L. Junkins, "Feedback control law for variable speed control moment gyroscopes," Journal of the Astronautical Sciences, vol. 46, no. 3, pp. 307-328, 1998.

[16] P. Tsiotras, "Stabilization and optimality results for the attitude control problem," Journal of Guidance, Control, and Dynamics, vol. 19, no. 4, pp. 772-779, 1996.

[17] H. Schaub and J. L. Junkins, "Stereographic orientation parameters for attitude dynamics: A generalization of the Rodrigues parameters," Journal of the Astronautical Sciences, vol. 44, no. 1, pp. 1-19, 1996.

[18] M. D. Shuster, "A survey of attitude representations," Journal of the Astronautical Sciences, vol. 41, no. 4, pp. 439-517, 1993.

[19] P. Tsiotras, H. Shen, and C. Hall, "Satellite attitude control and power tracking with energy/momentum wheels," Journal of Guidance, Control, and Dynamics, vol. 24, no. 1, pp. 23-34, 2001.

[20] H. Yoon and P. Tsiotras, "Singularity analysis of variable-speed control moment gyros," Jornal of Guidance, Control, and Dynamics, vol. 27, no. 3, pp. 374-386, 2004.

[21] R. Hebner, J. Beno, and A. Walls, "Flywheel batteries come around again,” IEEE SPECTRUM, vol. 39, pp. 46-51, Apr. 2002.

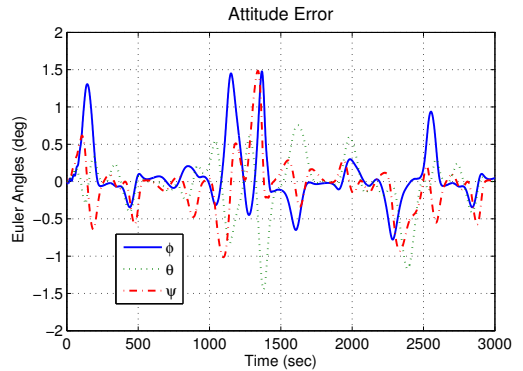

Fig. 2. Tracking Error Without Adaptation.

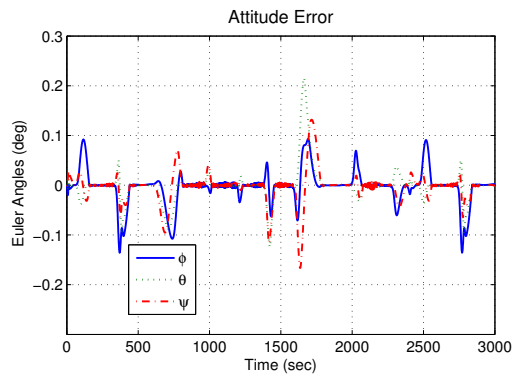

Fig. 3. Tracking Error With Adaptation of $\Theta_{a}$ Only.

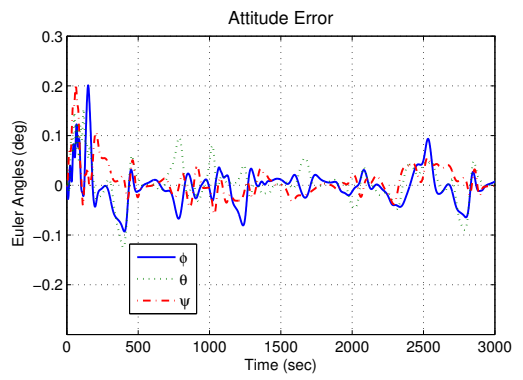

Fig. 4. Tracking Error With Adaptation of $\Theta_{s}$ Only.

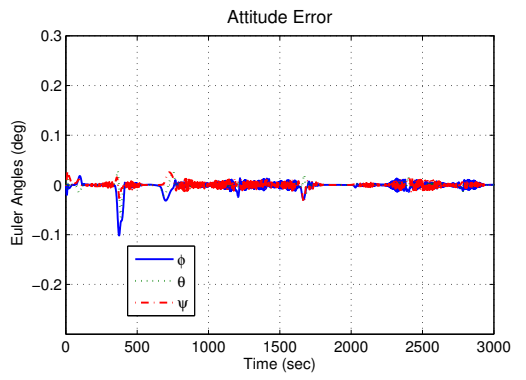

Fig. 5. Tracking Error With Adaptation of $\Theta_{a}$ and $\Theta_{s}$.

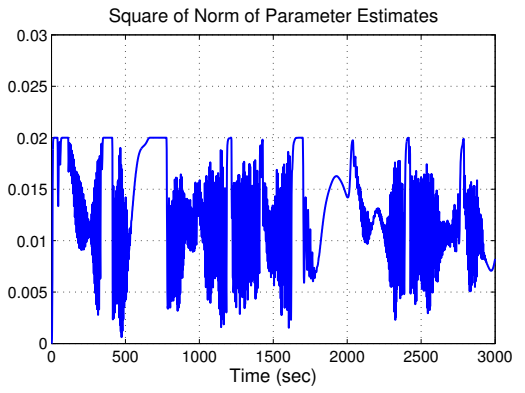

Fig. 6. Square of Norm of Parameter Estimate $\hat{\Theta}_{a}$. 